\title{
Dwarf spheroidal evolution: global view
}

\author{
Stefania Salvadori ${ }^{1}$, Andrea Ferrara ${ }^{1}$ and Raffaella Schneider ${ }^{2}$ \\ ${ }^{1}$ SISSA/International School for Advanced Studies, \\ via Beirut 4, 34100, Trieste, Italy \\ email: salvas@sissa.it \\ email: ferrara@sissa.it \\ ${ }^{2}$ INAF/Osservatorio Astrofisico di Arcetri, Largo Enrico Fermi 5, 50125, Firenze, Italy \\ email: raffa@arcetri.astro.it
}

\begin{abstract}
We propose a cosmological approach to investigate the formation and evolution of dwarf spheroidal galaxies, satellites of the Milky Way, which allows us to follow self-consistently the dSphs and MW formation, matching simultaneously most of their observed properties.
\end{abstract}

Keywords. cosmology: theory, galaxies: dwarf, galaxies: evolution, galaxies: formation, stars: Population II

\section{Introduction}

The formation and evolution of dwarf spheroidal galaxies (dSphs) is still a matter of debate despite the growing number of observational data collected. Recent observations (Helmi et al. 2006) of the stellar Metallicity Distribution Function (MDF) in four nearby dSphs, satellites of the Milky Way (MW), increase the number of unsolved questions pertaining to the origin of such puzzling objects. Conversely to expectations indeed, all the observed MDFs show a common cut-off at $[\mathrm{Fe} / \mathrm{H}] \sim-3$ and a lack of stars below such a value. On the contrary, the MDF of Galactic Halo stars, i.e. stars which reside into the same MW environment, point to a low $[\mathrm{Fe} / \mathrm{H}]$-tail which extends down to $[\mathrm{Fe} / \mathrm{H}] \sim-4$ (Beers \& Christlieb 2006) and even below (Christlieb et al. 2002, 2006; Frebel et al. 2005). We investigate the origin of such a discrepancy by analyzing the evolution of dSphs from a cosmological point of view.

\section{Building-up the MW}

We build-up the cosmological background by using the semi-analytical Monte Carlo code GAMETE (GAlaxy MErger Tree \& Evolution) which allows us to follow the star formation (SF) and chemical enrichment history of the MW along its hierarchical merger tree (Salvadori, Schneider \& Ferrara 2007, updated version in Salvadori, Ferrara \& Schneider 2008, hereafter SSF07 and SFS08).

The hierarchical merger history of the MW is reconstructed by decomposing the present-day Galactic dark matter halo into its progenitors running backward in time up to $z=20$. At any time-step haloes can either loose part of their mass (corresponding to a cumulative fragmentation into haloes below the resolution limit $M<M_{\text {res }}$ ) or loose mass and fragment into two progenitors. The mass below the resolution limit accounts for the Galactic Medium (GM) which represents the mass reservoir into which haloes are embedded and out of which they virialize and accrete mass; in other words it is the MW environment. We assume that feedback suppresses SF in mini-haloes and that only Ly $\alpha$ cooling haloes $\left(T_{v i r}>10^{4} \mathrm{~K}\right)$ contribute to the SF history (SFH) and chemical enrichment of the Galaxy. Consistently, we adopt a mass resolution $M_{\text {res }}=M_{4}(z) / 10$, 
where $M_{4}$ represents the halo mass corresponding to a virial equilibrium temperature $T_{\text {vir }}=10^{4} \mathrm{~K}$ at a given redshift $z$. The SF rate (SFR) is taken to be proportional to the mass of cold gas which increases according to an infall rate (Kereš et al. 2005) if haloes are newly virialized. Following the critical metallicity scenario (Bromm et al. 2001; Omukai 2000; Omukai et al. 2005; Schneider et al. 2002, 2003, 2006) we assume that low-mass $\mathrm{SF}$ is triggered by the presence of metals (and dust) exceeding $Z_{c r}=10^{-5 \pm 1} Z_{\odot}$. Indeed, massive Pop III stars can only form when the gas in the SF haloes has a metallicity $Z \leqslant Z_{c r}$; a typical mass value $m_{\text {popIII }}=200 M_{\odot}$ is assumed. Otherwise $\left(Z>Z_{c r}\right)$ Pop II/I stars form according to a Larson Initial Mass Function (IMF).

Once formed, stars contribute to the chemical enrichment of gas into both protoGalactic haloes (ISM) and GM, in its finite lifetime (i.e. the Instantaneous Recycling Approximation is relaxed). A mechanical feedback prescription (see SFS08 for details) describes the dispersion of metals into the GM driven by supernovae (SN) explosions. The gas ejection rate results $\dot{M}_{e j} \propto \dot{N}_{S N} / v_{e}^{2}$ where $\dot{N}_{S N}$ is the SN explosion rate and $v_{e}^{2}$ the escape velocity of the gas from the host halo. Consistently with previous studies (Vader 1986, Mac Low \& Ferrara 1999, Fujita et al. 2004) the ejected gas is assumed to be metal-enhanced with respect to star forming gas. Produced metals are instantaneously and homogeneously mixed with the gas, both in the ISM and in the GM.

\section{The Galactic Halo MDF}

The model is calibrated by using the available observations. The global properties of the MW (gas/stellar mass and metallicity) fix the efficiency of SF and SN wind independently of the adopted $Z_{c r}$ value. The Galactic halo MDF is used to constrain the latter parameter; the low- $[\mathrm{Fe} / \mathrm{H}]$ cut-off, in fact, is very sensitive to variations of $Z_{c r}$, being shifted to lower $[\mathrm{Fe} / \mathrm{H}]$ values when $Z_{c r}$ decreases (Fig. 1 ). The model $Z_{c r}=10^{-4} Z_{\odot}$ provides a very good fit to the MDF, including the peculiar cut-off observed at $[\mathrm{Fe} / \mathrm{H}]=-4$. However, it cannot account for the presence of three observed stars below $[\mathrm{Fe} / \mathrm{H}]<-4$ that can be explained by models with $Z_{c r} \leqslant 10^{-6} Z_{\odot}$ but at the price of overpopulating the $[\mathrm{Fe} / \mathrm{H}]<-4$ region (see SSF07 for details). In what follows, we will fix $Z_{c r}=10^{-4} Z_{\odot}$. Interestingly we found that most of the iron-poor stars which populated the Galactic

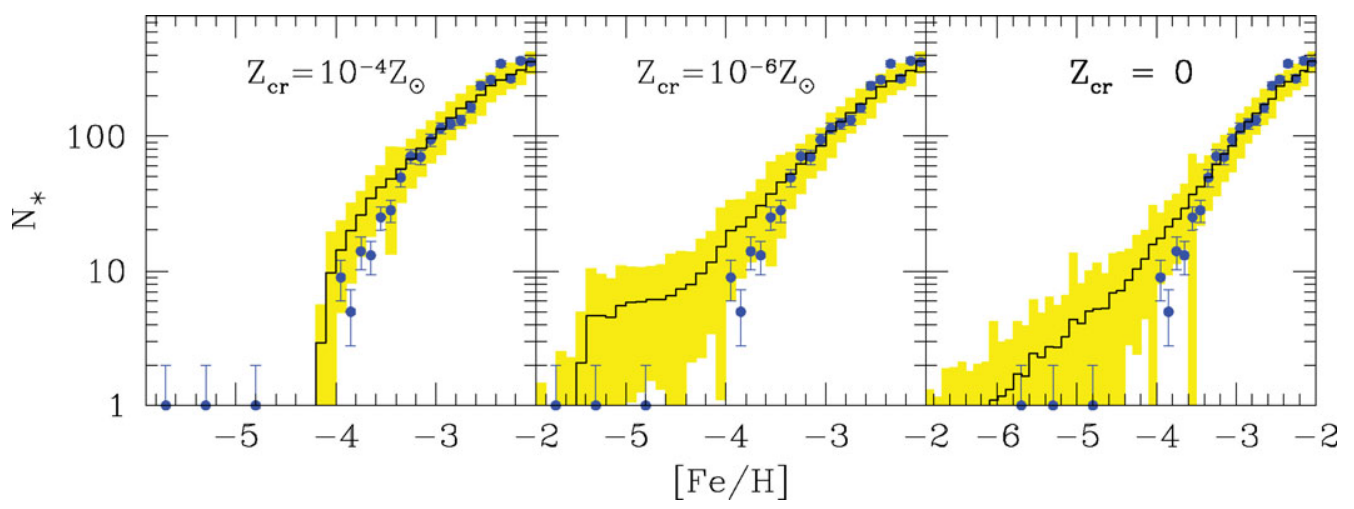

Figure 1. Comparison between the Galactic halo MDF observed by Beers \& Christlieb (private communication) with the inclusion of the three hyper-metal poor stars (Christlieb et al. 2002; Frebel et al. 2005) (points) and the simulated ones obtained by using the models $Z_{c r}=\left(10^{-4}, 10^{-6}, 0\right) Z_{\odot}$. The histograms are the average value of the MDFs over 200 realizations of the merger tree re-normalized to the number of observed stars with $[\mathrm{Fe} / \mathrm{H}] \leqslant-2$. The shaded area represents $\pm 1 \sigma$ Poissonian errors. 

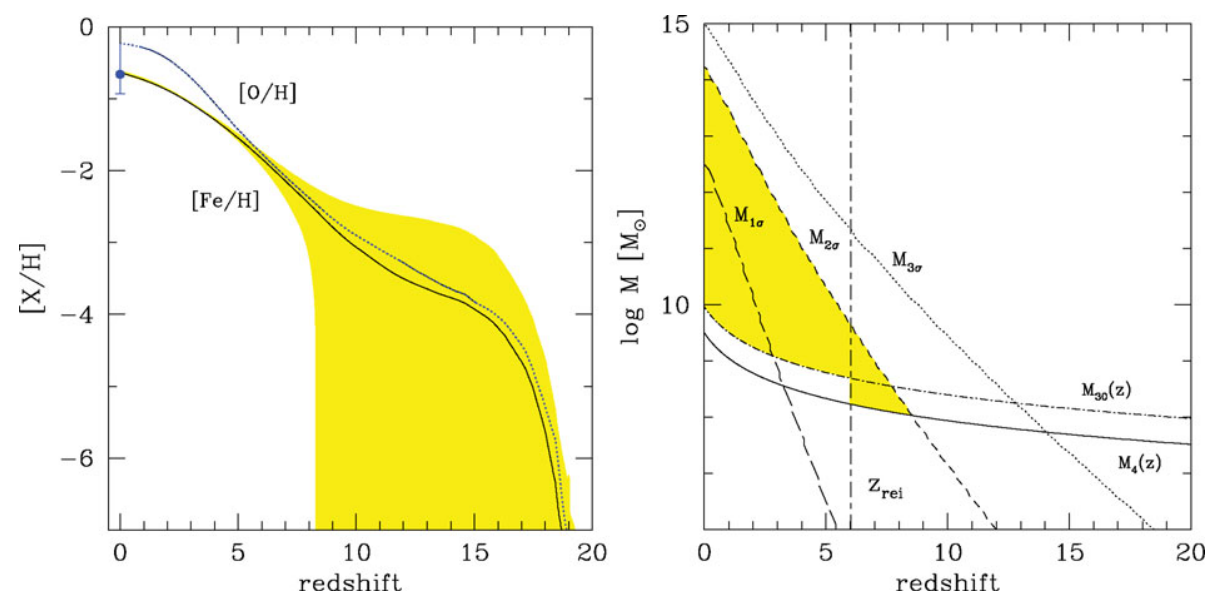

Figure 2. Left panel: Evolution of GM iron and oxygen (solid and dotted lines) abundance. Lines are the average values over 100 realizations of the merger tree and the shaded area the $\pm 1 \sigma$ dispersion region for $[\mathrm{Fe} / \mathrm{H}]$. The point is the measured $[\mathrm{O} / \mathrm{H}]$ in high-velocity clouds (Ganguly et al. 2005). Right panel: Evolution of the mass corresponding to $1 / 2 / 3 \sigma(M, z)$ density peaks (long-dashed/short-dashed/dotted lines); the solid and dotted-dashed lines show the evolution of $M_{4}(z)$ and $M_{30}(z)$ respectively. The selected reionization redshift $z_{r e i}=6$ is also shown.

halo $\mathrm{MDF}$ at $[\mathrm{Fe} / \mathrm{H}]<-2.5$ form in unpolluted haloes accreting Fe-enhanced gas from the GM. The initial $[\mathrm{Fe} / \mathrm{H}]$ abundance of the gas is then fixed by the corresponding GM abundance at the virialization redshift $\left(z_{v i r}\right)$. The evolution of the GM iron and oxygen abundance is shown in Fig. 2 (left panel).

\section{The dSph evolution}

We can now study the formation and evolution of dSphs inside our cosmological picture by assuming that dSph candidates belong to MW building blocks.

\subsection{Birth environment}

The first point to address is the selection criteria to identify dSphs among various MW progenitors. We select dSph candidates on the basis of their host halo mass using two criteria: one founded on reionization and the other on dynamical arguments. We already discussed (Sec. 2) that $M_{4}(z)$ is the minimum halo mass to trigger the SF; thus dSph candidates must have $M>M_{4}(z)$. During reionization, however, the increase of the Inter Galactic Medium (IGM) temperature causes the growth of the Jeans mass and consequent suppression of gas infall in low-mass objects. Essentially, below a characteristic halo massscale, the gas fraction is drastically reduced compared to the cosmic value (Gnedin 2000) and the SF consequently quenched. We adopt the simplest prescription assuming that, after reionization, SF is suppressed in haloes with circular velocity $v_{c}<30 \mathrm{~km} / \mathrm{s}$. Thus, when $z<z_{r e i}$ (here fixed at $z_{r e i}=6$ ), dSph candidates must have a mass $M_{30}(z)>$ $M\left(v_{c}=30 \mathrm{~km} / \mathrm{s}, z\right)$. Second, we want to select dSphs among virializing haloes which could become MW satellites. Following the results of N-body cosmological simulations by Diemand, Madau \& Moore (2005) we require that dSph candidates correspond to low- $\sigma$ density fluctuations at the virialization epoch $\left(z_{v i r}\right)$. In particular we select those objects with masses $M<M_{2 \sigma}$. Figure 2 (right panel) provides a summary of our selection criteria. Since the probability to form newly virializing haloes with $M>M_{30}$ is very low at each redshift, the first criterion implies that the formation of dSphs is unlikely 
to occur below $z_{r e i}$. Thus, dSph candidates are selected in a very narrow redshift range $6<z<8.5$. In this interval the mean GM iron abundance is $[\mathrm{Fe} / \mathrm{H}] \sim-3$ : the $\mathrm{dSph}$ birth environment has been naturally pre-enriched up to $[\mathrm{Fe} / \mathrm{H}]$ values consistent with those implied by observations of the MDFs by Helmi et al. (2006).

\subsection{Star formation history}

The dSph evolution is highly influenced by mechanical feedback effects, which are more intense in low mass objects (McLow \& Ferrara 2000, Ferrara \& Tolstoy 2002). The intermittent SFR (see Fig. 3 of SFS08) we derive for a typical dSphs is a consequence of such strong mechanical feedback processes. After $\sim 100$ Myr from the dSph formation the mass of gas lost by SN-driven winds becomes larger than the remaining gas into the galaxy, causing a complete blow-away of the gas. The dSph becomes gas free and the SF is stopped. At subsequent times, however, gas returned by evolved stars can be gradually collected: the dSph enters in a rejuvenation phase and the SF can start again. From the beginning of the rejuvenation phase the subsequent evolution of the galaxy proceeds like in the first $100 \mathrm{Myr}$ of its life although the SFR is more than 2 orders of magnitude lower due to the paucity of returned gas. As a consequence we found that the $99 \%$ of today living stars is formed during the first $100 \mathrm{Myr}$ of the dSph life, implying that dSphs are dominated by an ancient stellar population.

\section{Some observable properties of dSph}

In the following Sections we will compare our numerical results with some of the most relevant observations. Given the amount of available data, we take Sculptor as a reference dSph to compare with.

\subsection{The Color-Magnitude Diagram}

The Color-Magnitude Diagram (CMD) represents one of the best tools to study the SFH of a galaxy. Starting from our numerical results for a typical dSph (SFR, ISM metallicity evolution, IMF) we have computed the corresponding synthetic CMD using the publicly available IAC-STAR code by Aparicio \& Gallart (2004). A simple randomization procedure has been adopted in order to simulate observational errors into the synthetic CMD and compare numerical results with data (see SFS08 for details). Despite the simple procedure used and the IAC-STAR limitations $\left(Z_{m i n}>0.005 Z_{\odot}\right)$ we can observe that the match between theoretical and experimental points is quite good (Fig. 3 left panel).

\subsection{The Metallicity Distribution Function}

We can finally focus on the Sculptor MDF. In Fig. 3 (right panel) the observed (Helmi et al. 2006) and simulated MDFs are compared, the latter being normalized to the total number of observed stars (513). As can be inferred from the Figure, the model shows a good agreement with the observed $\mathrm{MDF}$, particularly for $[\mathrm{Fe} / \mathrm{H}]<-1.5$; a marginally significant deviation is present at larger $[\mathrm{Fe} / \mathrm{H}]$ values (see SFS08 for the discussion). From our analysis we found that infall rate controls the shape of the low- $[\mathrm{Fe} / \mathrm{H}] \mathrm{MDF}$ tail while mechanical feedback (and so differential winds) strongly affects the high- $[\mathrm{Fe} / \mathrm{H}]$ region. The two remaining free parameters of the models (infall and differential winds) are fixed in order to match the Sculptor MDF.

\section{Conclusions}

We proposed a cosmological approach to investigate the formation and evolution of dSph galaxies. In this scenario dSphs represent fossil objects that virialize at $z=7.2 \pm 0.7$ 

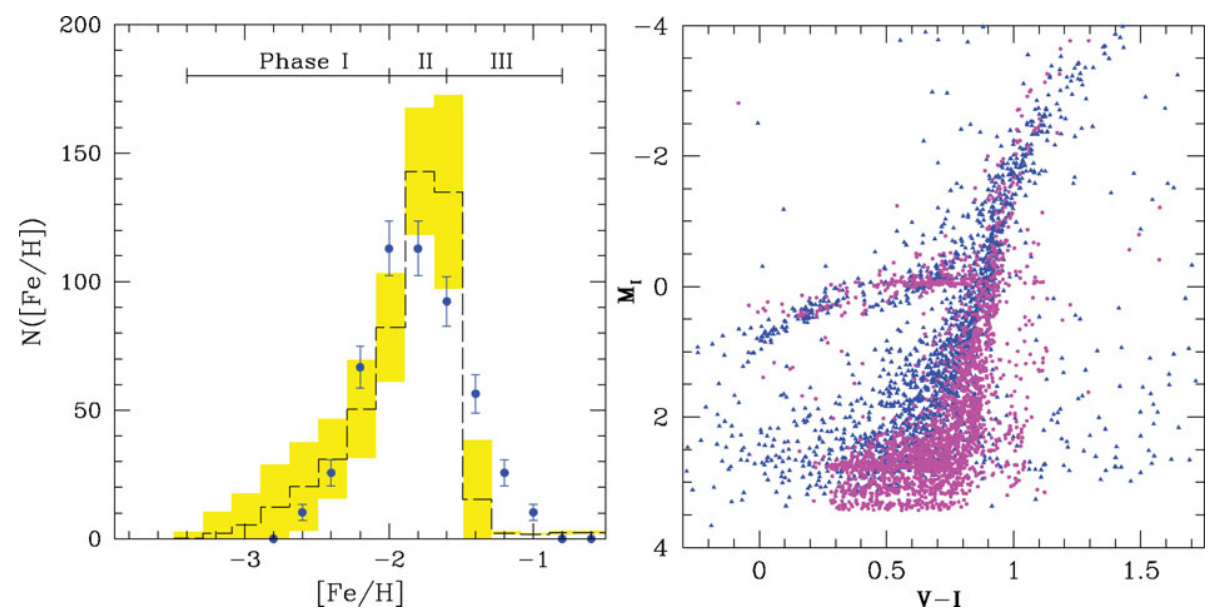

Figure 3. Left panel: Comparison between the observed (Helmi et al. 2006) (points) and simulated Sculptor MDF (histogram). Error bars are Poissonian errors. The histogram is averaged MDF over the surviving number of satellites $(\sim 20)$ in 100 realizations of the merger tree $(\sim 2000$ objects) and the shaded area the $\pm 1 \sigma$ dispersion region among different realizations. Right Panel: Comparison between the CMD of the Sculptor stellar population observed by Tolstoy et al. (2004) (triangles) and the synthetic CMD (open points) derived for a typical dSph galaxy with total mass $M=1.6 \times 10^{8} M_{\odot}$ which virialize at redshift $z_{v i r}=7.2$.

(i.e. in the pre-reionization era $z>z_{r e i}=6$ ) in the MW environment which at that epoch had already been pre-enriched up to $[\mathrm{Fe} / \mathrm{H}] \sim-3$. Their dynamical masses are in the narrow range $M=(1.6 \pm 0.7) \times 10^{8} M_{\odot}$. Our results match several observed properties of Sculptor, used as a template of dSphs: (i) the Metallicity Distribution Function; (ii) the Color Magnitude Diagram; (iii) the decrement of the stellar [O/Fe] abundance ratio for $[\mathrm{Fe} / \mathrm{H}]>-1.5$; (iv) the dark matter content and the light-to-mass ratio; (v) the HI gas mass content.

\section{References}

Bromm, V., Ferrara, A., Coppi, P. S., \& Larson, R. B., 2001, MNRAS, 328, 969

Christlieb, N., et al. 2002, Nature, 419, 904

Frebel, A., Christlieb, N., Norris, J. E., Aoki, W., \& Asplund, M. 2005, Nature, 434, 871

Fujita, A., Mac Low, M. M., Ferrara, A., \& Meiksin, A., 2004, ApJ, 613, 159

Ganguly, R., Sembach, K. R., Todd, T. M., \& Savage B. D. 2005, ApJ, 157, 251

Helmi, A., et al. 2006, ApJ, 651, L121

Kereš, D., Katz, N., Weinberg, D. H., \& Davé, R., 2005, MNRAS, 363, 2

Mac Low, M.-M. \& Ferrara, A. 1999, ApJ, 513, 1421

Omukai, K., 2000, ApJ, 534, 809

Omukai, K., Tsuribe, T., Schneider, R., \& Ferrara, A., 2005, ApJ, 626, 627

Salvadori, S., Schneider, R., \& Ferrara, A. 2007, MNRAS, 381, 647

Salvadori, S., Ferrara, A., \& Schneider, R. 2008, MNRAS, 381, 647

Schneider, R., Ferrara, A., Natarajan, P., \& Omukai, K., 2002, ApJ, 571, 30

Schneider, R., Ferrara, A., Salvaterra, R., Omukai, K., \& Bromm V., 2003, Nat, 422, 869

Schneider, R., Omukai, K., Inoue, A. K., \& Ferrara, A., 2006, MNRAS, 369, 1437

Tolstoy, E., et al. 2004, ApJ, 617, L119

Vader, J. P., 1986, ApJ, 305, 669 